\title{
CO2 Reduction at Low Overvoltage in Neutral Aqueous Solution by Ni-cyclam Attached Polyallylamine Polymer
}

\author{
Woonsup Shin*, D. Saravanakumar, Jieun Song, Nayoung Jung, \\ and Harishchandra Jirimali \\ Department of Chemistry, Interdisciplinary Program of Integrated Biotechnology, and \\ Korea Center for Artificial Photosynthesis, Sogang University, Seoul 121-742, Korea(shinws@sogang.ac.kr)
}

Artificial photosynthesis comprise of a light-driven water oxidation reaction and an electrochemical $\mathrm{CO}_{2}$ reduction in dark. To achieve the efficient $\mathrm{CO}_{2}$ reduction by the excited electron from the light-driven anodic reaction, the lowering the overvoltage of $\mathrm{CO}_{2}$ reduction is an essential component. [Ni(cyclam) ${ }^{2+}$ (cyclam=1,4,8,11-tetraazacyclotetradecane) and its derivatives are known to be efficient in $\mathrm{CO}_{2}$ to $\mathrm{CO}_{\text {conversion }}$ and the macrocyclic ligand, cyclam is known to stabilize Ni(I) state. However, those compounds still have ca. 0.6 $\mathrm{V}$ of overvoltages and the use of mercury electrode is essential to suppress hydrogen evolution on the electrode at such a negative potential. We found that the incorporation of the Ni(cyclam) unit into polyallylamine (PALA) backbone by Schiff' $s$ base condensation via axial coordination of 4-pyridine carboxaldehyde is effective in lowering overvoltage and it enables the catalyst to be used with carbon electrode material in neutral aqueous solution. The resulting redox polymer, Ni(cyclam)-PALA converts $\mathrm{CO}_{2}$ to $\mathrm{CO}$ near its thermodymic potential, -0.78 $\mathrm{V}$ vs. $\mathrm{Ag} / \mathrm{AgCl}$ in $\mathrm{pH} 8,50 \mathrm{mM}$ tris buffer on glassy carbon electrode with current efficiency of over $90 \%$. The axial coordination of 4-pyridine unit is mainly responsible for lowering the overpotential and the attached polyallylamine partly helps.

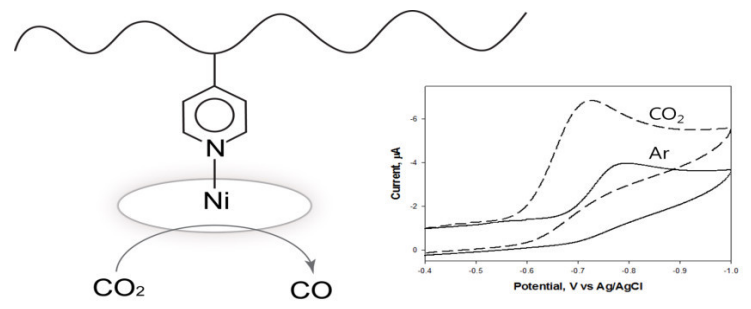

\section{References}

1. Saravanakumar, D.; Song, J.; Jung, N.; Jirimali, H.; Shin, W. ChemSusChem 2012 5, 634. 